\title{
Preliminary design issues for inertial rings in Ambient Assisted Living applications
}

\author{
Dario Esposito, Filippo Cavallo \\ The BioRobotics Institute \\ Scuola Superiore Sant'Anna \\ Pontedera (Pisa), Italy \\ \{d.esposito; f.cavallo\}@sssup.it
}

\begin{abstract}
A wearable 9dof inertial system able to measure hand posture and movement is presented. The design issues for the deployment of measurement instrumentation based on noinvasive ring-shaped inertial units and of a wireless sensor network by them composed are described. Compromises between the physical and functional proprieties of a wearable device and the requirements for the hardware development are discussed with attention to an handsome design concept aesthetically effective. Techniques of power saving based on an optimized firmware programming are mentioned to realize a performing battery powered system featured by an exhaustive operation time. The printed circuit board (PCB) design rules, the choice of the components and materials, the fusion of inertial data with optical sensors outcomes are also discussed. Previous experience in the field of wearable systems are mentioned in the presentation of the results that emphasize the functional and application potential of a 9dof inertial system integrated in a ring-shaped device.
\end{abstract}

Keywords - Wearable technology; inertial sensors; power management; hand movement analysis.

\section{INTRODUCTION}

The rising demand for sustainable healthcare systems, the increasing aging population, and the longer retirement have increased the importance of Ambient Assisted Living (AAL) developments, services, and products [1]. Nowadays the telecare and telehealth markets are the most development ones and, indeed, the home healthcare industry is testing telehomecare and tele-monitoring services that represent a valuable opportunity to balance quality of care with cost control. By using telephone, satellite, and Internet-based communication, these services allow medical care professionals to stay in touch with patients without travelling to the patients' homes. In this context the wearable technologies play an important role in supporting medical staff in diagnosis, therapy and monitoring of patients [2]. It is well-known how the number of the wearable devices sold is globally increasing (nearly tripled in between 2011-2012) with 8.3 million of fitness trackers, smart watches and other connected wearable bought by consumer in 2012 [3]. Worldwide spending on wearable technology was 1.4 billion in 2013 [4], but 2014 is, for sure, the year of the wearable tech highlighted by the attention paid to this sector of the market by all the most important industrial realities; a growing number of devices have been launched on the market and many are in development to offer new and disparate functionalities in the short-term : smart watches, smart glasses, as well as, smart clothes. Market analysis and intelligence agency ABI Research predicts that this year, about 90 million wearable devices will be sold and demand will be higher in 2015 [5] ; most of the gadgets are believed to be related to health and fitness. According to the research firm Juniper by 2018 this market will be worth $€ 15$ billion for more than 130 million units sold[4].

This work presents the design issues to deal with in the development of a wireless sensorised wearable device ringshaped based on inertial and optical sensors able to perform a long-term hand posture and movement analysis.

There are several studies concerning the implementation of wearable devices mainly glove-shaped used to analyze and faithfully reproduce the movement of the hand for different purposes. Some of these devices are only prototypes, others are commercial products[6-7]. The technologies most recurrent are based on flex/force sensors [8-9], inertial sensors [10]-[15] or on their combination [16]; these devices belong to the field of the biomedical instrumentation aimed at the assistance of patients with movement disorders and disabilities [17], but also as means of human-robot/machine interaction or as hardware controllers through methods of gesture recognition [18]. First academic studies regarding sensorized wearable rings are emerged [19] and new small companies composed by team of engineers, scientist, product managers, $\mathrm{PhD}$ and master recipients are investing in the wearable tech, putting in presale devices of various kinds, including rings equipped with sensors [20].

The feasibility studies of a 9dof inertial system integrated in a wearable device ring-shaped are a step ahead of a work carried out over the time on the study of Parkinson's disease[21]-[23] that leads to an European patent application [24]. In this previous work the proper and suitable features of inertial wired rings structured in a glove-type device have been validated for the measurement of biomechanical parameters aimed at the monitoring of the disease and at the formulation of a new method of early diagnosis. The desire to break down the structural limitations tied to a wired device led to the development of the prototypal version submitted and to the guidelines described with the aim to overcome the issues addressed specially in terms of circuital integration and reduction of the power consumptions. Possible guidelines focused on the implementation of a sensorized ring equipped with commercial components are now presented; attention is 
given to issues related to the PCB layout and design concept and, in particular, to the slaughter of energy consumptions.

\section{INSTRUMENTS}

The system is composed of two kinds of elements, one bracelet and up to five rings, one for each finger. The bracelet and the five rings communicate by means of a wireless sensor network, based on the Bluetooth Piconet Stack. The bracelet acts as the master of the Piconet, while the rings are conceived to be slave devices.

The bracelet is designed with the microcontroller (mcu) and the SPBT2632C2A Bluetooth module, both from STMicroelectronics, Italy, and includes also a complete 9DOF inertial measurement unit (LSM303DLHC - accelerometer and magnetometers, L3G4200D - gyroscope). The inertial measurement unit includes triaxial 16-bit accelerometers, gyroscope and magnetometer factory calibrated.

Each ring, instead, is composed of the same components of the bracelet and, additionally, of two other sensors: a digital proximity, ambient light, RGB, gesture detection sensor (APDS9960, Avago Technologies) and an analog reflective object sensor (QRE1113GR, Fairchild Semiconductor). Both these sensors are integrated in the rings to easily discriminate ambiguities of the measure, to establish specific thresholds and to expand the sensory capabilities of the device.

The choice of the components derived from a comparison with similar commercial products in terms of linearity, measurement repeatability and possible drifts of the data but also considering power consumptions and packages in terms of shapes and footprint encumbrance.

The bracelet and rings are powered by two different batteries. Because of the limited space on the fingers, rings are powered by a miniaturized battery of $25 \times 15 \times 3 \mathrm{~mm}$ and $100 \mathrm{mAh}$. Instead, since a larger battery could be installed in the bracelet, a battery of $48 \times 30 \times 6 \mathrm{~mm}$ and $800 \mathrm{mAh}$ is used. Both the devices are equipped with an embedded battery charger and a system to automatically detect the presence of an external DC supply.

Inertial sensor signals are acquired at an fs frequency rate and then low pass filtered at $5 \mathrm{~Hz}$ through a real time IIR filter implemented on each microcontroller.

Each one of the ring units represents a node of a local Bluetooth Piconet, which establishes a coordinated and synchronized Wireless Sensor Network (WSN) in correspondence of the hand. The coordinator of the network, which fills the role of the master, could be one of the rings or an external unit located, for example, on the wrist embedded in a thin bracelet.

The wrist module could ensure the measure of the motion related to the forearm equipping it with a IMU but also the possibility to incorporate a micro SD card to store data or radio modules based on different protocol of communications such as Wi-Fi or ZigBee to transmit data towards different control stations (i.e. pc station ,tablet, notebook, smartphone and smartTV).
In Fig. 1 it is presented a first prototypal version of the ring necessary to test on-lab the proper electrical and functional features of the device confirming the correctness of the schematics realized through appropriate cad software.

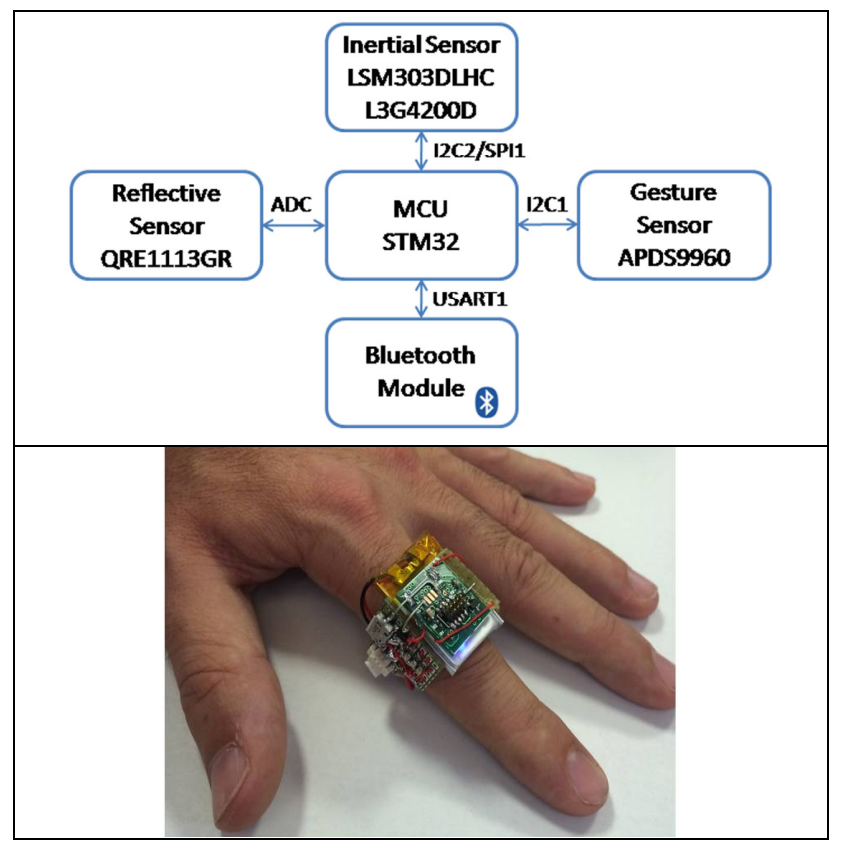

Fig.1- First prototype of the ring, worn on the finger with battery attached.

\section{METHODS}

The rings are the most critical part of the system, because, due to the limited space and the requirement of miniaturization, they cannot be endowed with long life batteries. Therefore appropriate design strategies are the basis of this approach. Here the present work focuses on one single ring, knowing that the same approach is to be considered also for all other units. The key design points are the following:

- Development of a soft circuit to be embedded in a ringshape chassis, appropriately designed;

- Implementation of ad-hoc firmware on the microcontroller, for optimized power management.

\section{PCB LAYOUT AND DESIGN CONCEPT}

The printed circuit board (PCB) layout of the electronic board was developed jointly with the design concept of a possible final ring prototype. The choice of the components, of the geometries, the number of layers and materials of the PCB was made according to the constraints imposed by the realization of a package both resistant and of minimum bulk such as to make the ring actually wearable, easy to use and comfortable. In particular, a four layer PCB was drafted addressing the typical design rules of a multilayer board placing two signal layers (TOP, BOTTOM), an inner ground and supply plane; appropriate values of insulation were setting in particular below the antenna area of the radio module to not break down the signal strength due to unwanted shielding. The minimum value of clearance between objects in the layers was set to 6 mils while the minimum drill was set to 11 mils: these parameters are the most stringent constraints depending on the 
technologies used for printing. The width of the electrical paths was assigned to 8 mils for the signal wires and to 10 mils for the ground and supply conductors but nothing would prevent to reduce the size of these paths in case of routing necessities whereas the maximum current values.

The board was designed thinking to a physical implementation in flexible (i.e. Polyimide, Mylar, Kapton or Teflon) or flex-rigid material (rigid parts connected by flexible sections) to incorporate the circuit inside the ring. Considering a flexible board it was divided into several sections; some of these were free of components, able to support a high curvature radius, with a length to be assigned obtaining different sizes of the rings. Other sections contained the components, they had assigned dimensions and they had to be positioned in the package with a small curvature radius such as to avoid breakage of the soldering pads.

In Fig. 2 is presented a cad project realized exploiting the CadSoft EAGLE PCB Design Software respecting the design rules described and using the footprint of the components chosen with the addition of command buttons, status leds and a micro-B type USB port.

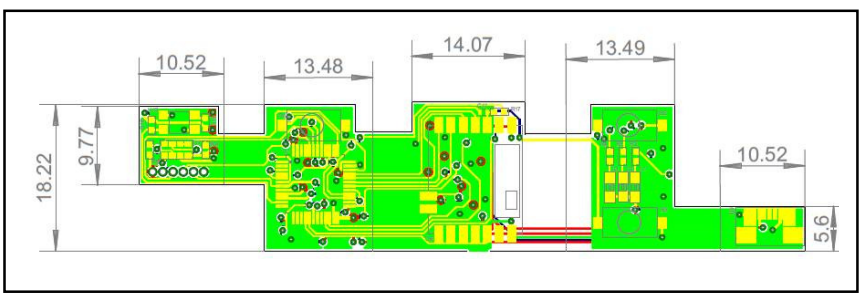

Fig.2- PCB layout realized through CadSoft EAGLE.

The measures of the fixed and symmetrical sections are expressed in millimeters. The proper positioning of the components and the shape of board is the consequence of a continuous update and optimization simultaneously to the formulation and design of the package; in Fig. 3 there is an example of a preliminary CAD design concept of the system.

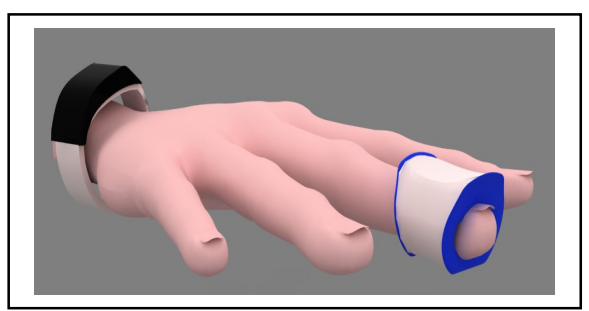

Fig 3 - preliminary CAD design concept of the system.

\section{RESUlts}

The power consumption of the ring-shaped device was measured extrapolating average and standard deviation (to highlights any current spikes) from the acquisition of 30 measures, sampling at $2 \mathrm{~Hz}$ with an AMPROBE bench multimeter. Without any kind of energy saving trick, the consumption was about $100 \mathrm{~mA}$ (i.e. 1 hour of working) with a data sampling frequency and radio transmission of $100 \mathrm{~Hz}$. The high speed external clock signal (HSE) was selected as system clock source equipping the microcontroller with a
$8 \mathrm{MHz}$ external oscillator. The internal phase-locked loop (PLL) was enabled fixing the mcu frequency at $72 \mathrm{MHz}$ (i.e. the maximum frequency allowed) programming the Reset and Clock Control registers (RCC) as shown in the source code in Fig.4:

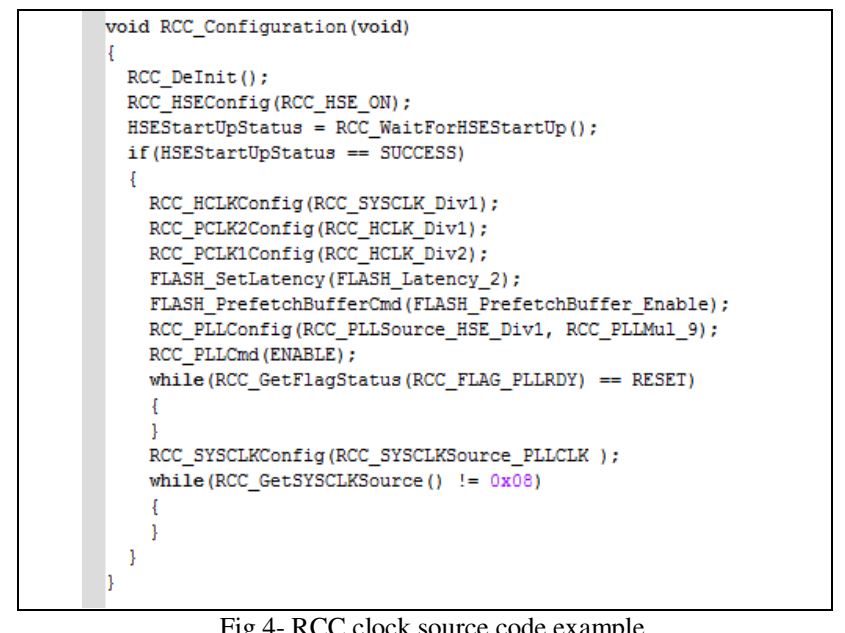

The communication interfaces of the microcontroller utilized to communicate with the digital sensors (SPI, I2C) and the I/O ports were set at the maximum speed allowed by the peripherals $(\mathrm{I} 2 \mathrm{C}$ at $400 \mathrm{KHz}$, SPI baud rate prescaler at 256, GPIO speed at 50MHz). The Universal Synchronous /Asynchronous Receiver/Transmitter protocol (USART) used to communicate with the radio module was initially set at 460800 bps.

In Tab. 1 are reported the consumptions in case of different sampling frequencies and radio baud rates:

Tab.1- Consumptions at different sampling frequencies and radio baud rates.

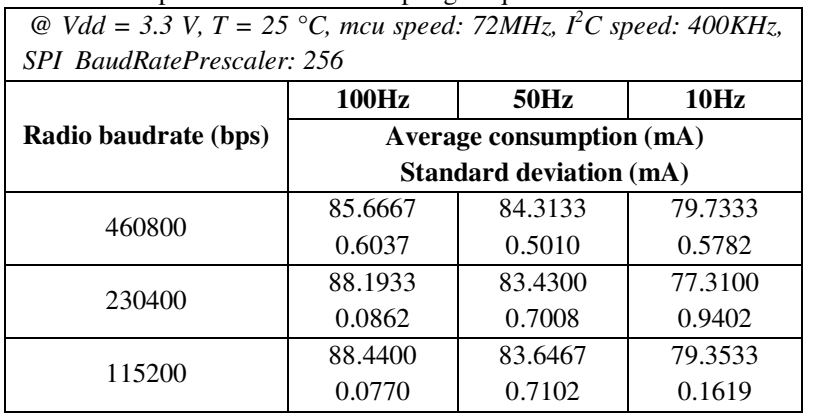

The chosen working point was represented by the combination of a sampling frequency of $50 \mathrm{~Hz}$ and a baud rate of 230400 bps exhaustive for the transmission of the data packet. The sampling frequency was generated by a general purpose timer (TIMx, with $\mathrm{x}>1$ ) and it was within its interruption handler (i.e. TIM2_IRQHandler) that the communication with sensors, data acquisition and transmission were possible.

An important observance is to avoid, in these types of 
applications, the use of the advanced control timer (TIM1) due to its higher current consumption $(1.8 \mathrm{~mA})$ in comparison with the general purpose timers $(1.2 \mathrm{~mA})$.

Digital components (microcontroller, inertial and gesture sensor) were subsequently programmed in sleep-wake up mode; to inhibit the analog reflective sensor it was necessary the addition of a logic NAND to manage its supply. The energy savings due to the power management of the microprocessor and of each sensor are presented in Tab. 2:

Tab.2- Power consumptions setting the sleep-wake up mode at $50 \mathrm{~Hz}$ sampling frequency.

\begin{tabular}{|c|c|}
\hline \begin{tabular}{|c|} 
@ Vdd $=3.3 \mathrm{~V}, \mathrm{~T}=2{ }^{\circ} \mathrm{C}, \mathrm{mcu}$ speed: $72 \mathrm{MHz}, \mathrm{I}^{2} \mathrm{C}$ speed $: 400 \mathrm{KHz}, \mathrm{SPI}$ \\
BaudRatePrescaler: 256, radio baud rate: 230400 bps, sampling \\
frequency:50Hz
\end{tabular} \\
\hline \multirow{2}{*}{ Sleep-wake up mode } & $\begin{array}{c}\text { Average consumptions (mA) } \\
\text { Standard deviation (mA) }\end{array}$ \\
\hline \multirow{2}{*}{ STM32F103REY6TR } & 74.2127 \\
& 1.0097 \\
\hline \multirow{2}{*}{ LSM303DLHC } & 79.3433 \\
& 0.1329 \\
\hline \multirow{2}{*}{ L3G4200D } & 82.2100 \\
& 1.1471 \\
\hline \multirow{2}{*}{ APDS9960 } & 86.1300 \\
& 0.1484 \\
\hline \multirow{2}{*}{ QRE111GR } & 74.2933 \\
& 1.1103 \\
\hline
\end{tabular}

Only the APDS9960 showed an increase in power dissipation due to its slow transient; at $10 \mathrm{~Hz}$ instead the sleepwake up mode showed satisfactory results and the final choice was to work at this sampling frequency with both the optical sensors and at $50 \mathrm{~Hz}$ with the inertial 9dof sensors. The consumptions at $10 \mathrm{~Hz}$ of the optical sensors are reported in Tab.3 for each sensor:

Tab.3- Power consumptions setting the optical sensors in sleep-wake up mode at $10 \mathrm{~Hz}$ sampling frequency.

@ Vdd $=3.3 \mathrm{~V}, \mathrm{~T}=25^{\circ} \mathrm{C}, \mathrm{mcu}$ speed: $72 \mathrm{MHz}, \mathrm{I}^{2} \mathrm{C}$ speed $: 400 \mathrm{KHz}, \mathrm{SPI}$

BaudRatePrescaler: 256 , radio baud rate:230400 bps, inertial sensors @ 50Hz,opticalsensors@ $10 \mathrm{~Hz}$

\begin{tabular}{|c|c|}
\hline Sleep-wake up mode (10Hz) & $\begin{array}{c}\text { Average consumptions (mA) } \\
\text { Standard deviation }(\mathbf{m A})\end{array}$ \\
\hline \multirow{2}{*}{ APDS9960 } & 80.2433 \\
& 0.2108 \\
\hline \multirow{2}{*}{ QRE111GR } & 72.6167 \\
& 0.6670 \\
\hline
\end{tabular}

The consumption of the entire system in sleep-wake up mode is presented in Tab.4:

Tab.4- Power consumptions of the entire system in sleep-wake up mode. @ Vdd $=3.3 \mathrm{~V}, \mathrm{~T}=25^{\circ} \mathrm{C}, \mathrm{mcu}$ speed: $72 \mathrm{MHz}, \mathrm{I}^{2} \mathrm{C}$ speed : $400 \mathrm{KHz}, \mathrm{SPI}$ BaudRatePrescaler: 256, radio baud rate:230400 bps, inertial sensors @ 50Hz,opticalsensors @ $10 \mathrm{~Hz}$

\begin{tabular}{|c|c|}
\hline \multirow{2}{*}{ Sleep-wake up mode } & $\begin{array}{c}\text { Average consumptions (mA) } \\
\text { Standard deviation (mA) }\end{array}$ \\
\hline \multirow{2}{*}{ All } & 50.5733 \\
& 0.6565 \\
\hline
\end{tabular}

To further reduce the power expenditure the Real Time Clock (RTC) and the External interrupt/event controller (EXTI) registers were properly programmed to generate a $50 \mathrm{~Hz}$ alarm interrupt sourced by the internal $32 \mathrm{KHz}$ lowpower source clock (LSI); in this way it was possible to manage the data acquisition/transmission through the RTC handler (RTCAlarm_IRQHandler) instead of a TIMx interrupt reconfiguring the internal $8 \mathrm{MHz}$ oscillator (HSI) as system clock source. The PLL was again enabled but forcing the mcu speed at a lower frequency of $12 \mathrm{MHz}$ as shown in Fig.5:

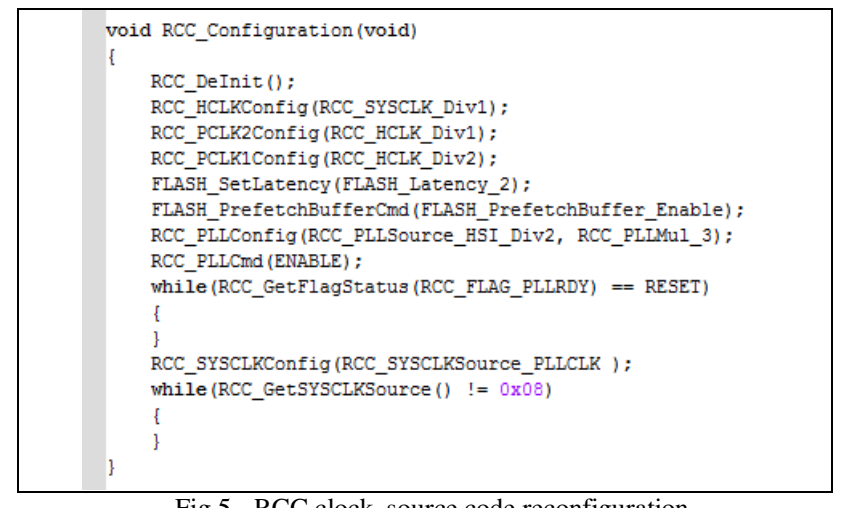

Fig 5 - RCC clock source code reconfiguration.

This arrangement resulted in a reduction of the consumption as reported in Tab.5:

Tab.5- Power consumption exploiting the RTC control features. @ Vdd $=3.3 \mathrm{~V}, \mathrm{~T}=25^{\circ} \mathrm{C}, \mathrm{mcu}$ speed: $12 \mathrm{MHz}, \mathrm{I}^{2} \mathrm{C}$ speed $: 400 \mathrm{KHz}, \mathrm{SPI}$ BaudRatePrescaler: 256, radio baud rate:230400 bps, inertial sensors @ 50Hz, optical sensors @ $10 \mathrm{~Hz}$, all components in sleep-wake up mode

\begin{tabular}{|c|c|}
\hline System clock reconfiguration & $\begin{array}{c}\text { Average consumptions (mA) } \\
\text { Standard deviation }(\mathbf{m A})\end{array}$ \\
\hline \multirow{2}{*}{ RTC, EXTI, RCC } & 38.7400 \\
& 1.1990 \\
\hline
\end{tabular}

The next step was to act on the peripheral clock gating which consists in a management of the APB/AHB busses enabling/disabling the clock peripherals at run time with the reduction of the power expenditure shown in Tab.6:

Tab.6 - Power consumption exploiting the peripheral clock gating: @ $V d d=3.3 \mathrm{~V}, \mathrm{~T}=25^{\circ} \mathrm{C}$, mcu speed: $12 \mathrm{MHz} \mathrm{I}^{2} \mathrm{C}$ speed $: 400 \mathrm{KHz}$, SPI BaudRatePrescaler: 256, radio baud rate:230400 bps, inertial sensors@50Hz,optical sensors @10Hz, all components in sleep-wake up mode

\begin{tabular}{|c|c|}
\hline Peripheral clock gating & $\begin{array}{c}\text { Average consumptions }(\mathbf{m A}) \\
\text { Standard deviation }(\mathbf{m A})\end{array}$ \\
\hline \multirow{2}{*}{$\mathrm{APB} / \mathrm{AHB}$} & 37.7333 \\
& 0.6857 \\
\hline
\end{tabular}

A further improvement was to reduce the SPBT2632C2A MCU speed from the default $32 \mathrm{MHz}$ to $8 \mathrm{MHz}$ clock, which was satisfactory to transmit the data packets. The AT command necessary to configure the Bluetooth module is 
reported in Fig.6:

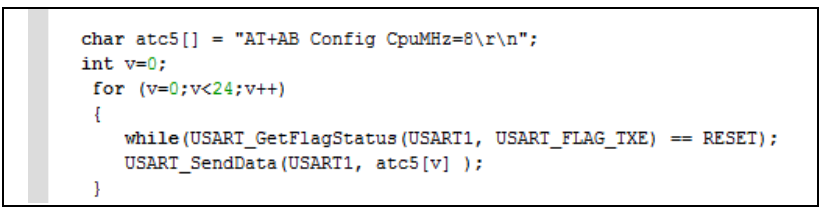

Fig 6 - AT command to program the SPBT2632C2A mcu speed

The relative power saving of this solution is instead reported in Tab.7:

Tab.7 - Power consumption with the SPBT2632C2A cpu speed at $8 \mathrm{MHz}$. @ $V d d=3.3 \mathrm{~V}, \mathrm{~T}=25^{\circ} \mathrm{C}$, mcu speed: $12 \mathrm{MHz}, \mathrm{I}^{2} \mathrm{C}$ speed $: 400 \mathrm{KHz}, \mathrm{SPI}$ BaudRatePrescaler: 256, radio baud rate:230400 bps, inertial sensors @ 50Hz, optical sensors @ $10 \mathrm{~Hz}$, all components in sleep-wake up mode

\begin{tabular}{|c|c|}
\hline Radio mcu speed at 8MHz & $\begin{array}{c}\text { Average consumptions (mA) } \\
\text { Standard deviation }(\mathbf{m A})\end{array}$ \\
\hline SPBT2632C2A & 32.6467 \\
& 2.0212 \\
\hline
\end{tabular}

The addition of an external $32,768 \mathrm{kHz}$ LPO allowed to force the SPBT2632C2A mcu in deep-sleep mode through the AT commands in Fig.7 :

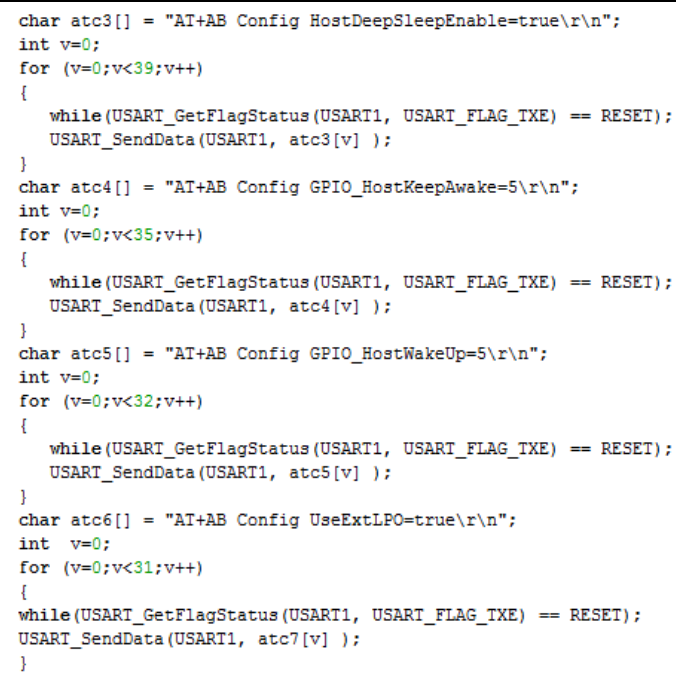

Fig 7 - AT command to program the SPBT2632C2A in deep-sleep mode.

The power consumption decreased at the value in Tab.8:

Tab.8 - Power consumption with the SPBT2632C2A in deep-sleep: @ $\mathrm{Vdd}=3.3 \mathrm{~V}, \mathrm{~T}=25^{\circ} \mathrm{C}$, mcu speed: $12 \mathrm{MHz}, \mathrm{I}^{2} \mathrm{C}$ speed $: 400 \mathrm{KHz}$, SPI BaudRatePrescaler: 256, radio baud rate: $230400 \mathrm{bps}$, mcu radio speed at $8 \mathrm{MHz}$ inertial sensors @ 50Hz, optical sensors @ $10 \mathrm{~Hz}$, all components in sleep-wake up mode

\begin{tabular}{|c|c|}
\hline Radio in deep-sleep & $\begin{array}{c}\text { Average consumptions (mA) } \\
\text { Standard deviation }(\mathbf{m A})\end{array}$ \\
\hline \multirow{2}{*}{ SPBT2632C2A } & 25.9267 \\
& 1.0834 \\
\hline
\end{tabular}

The last optimization consisted in the reduction of the
SPBT2632C2A baud rate at 115200 bps (considering the carried out reduction of the radio mcu speed) which led to the power consumption in Tab.9:

Tab.9 - Power consumption with the radio baud rate at $115200 \mathrm{bps}$ : (a) $\mathrm{Vdd}=3.3 \mathrm{~V}, \mathrm{~T}=25^{\circ} \mathrm{C}$, mcu speed: $12 \mathrm{MHz}, \mathrm{I}^{2} \mathrm{C}$ speed : $400 \mathrm{KHz}, \mathrm{SPI}$ BaudRatePrescaler: 256, mcu radio speed at $8 \mathrm{MHz}$ and deep-sleep mode, inertial sensors @ 50Hz, optical sensors @10Hz, all components in sleep-wake up mode

\begin{tabular}{|c|c|}
\hline Radio baud rate at $\mathbf{1 1 5 2 0 0}$ bps & $\begin{array}{c}\text { Average consumptions }(\mathbf{m A}) \\
\text { Standard deviation }(\mathbf{m A})\end{array}$ \\
\hline SPBT2632C $2 \mathrm{~A}$ & 19.2733 \\
& 1.9496 \\
\hline
\end{tabular}

The total energy savings achieved through this firmware optimization was about $64 \mathrm{~mA}$ ( $70 \%$ of the initial value) with $50 \mathrm{~Hz}$ data transmission, $50 \mathrm{~Hz}$ inertial sensors sampling frequency and $10 \mathrm{~Hz}$ optical sensors one.

\section{DISCUSSION AND CONCLUSION}

The work presented wants to deal with some design issues for the implementation of a miniaturized wearable device on the basis of technological resources made available by the components on the market. The realization of a package aesthetically valid and which allows wearing the device without impeding the free movement of the fingers, especially in the case of network, imposes stringent constraints on the hardware to be integrated.

The choice of components must be born from a compromise between performance and available package because even small differences in the footprint can have a significant weight for the realization of electronic tracks.

The size of the battery and its housing are the strongest constraint from having to deal with hence the need to limit the energy consumption as much as possible.

The continuous research for the design of ever more efficient batteries and, at the same time, even more compact, of new materials (e.g. flexible battery type) for their physical implementation can definitely lead towards a technological improvement in the performance of the ring-shaped device.

In this sense, a possible future development, maintaining the same components, could consist in the optimization of the radio module transmission putting it in sniff mode operation; sniff mode consists in periodic intervals in which the radio is forced to exit the stage of deep-sleep to transmit or receive.

An ensemble of AT2 command to proper configure the Bluetooth module in sniff mode are listed in Fig.7: 


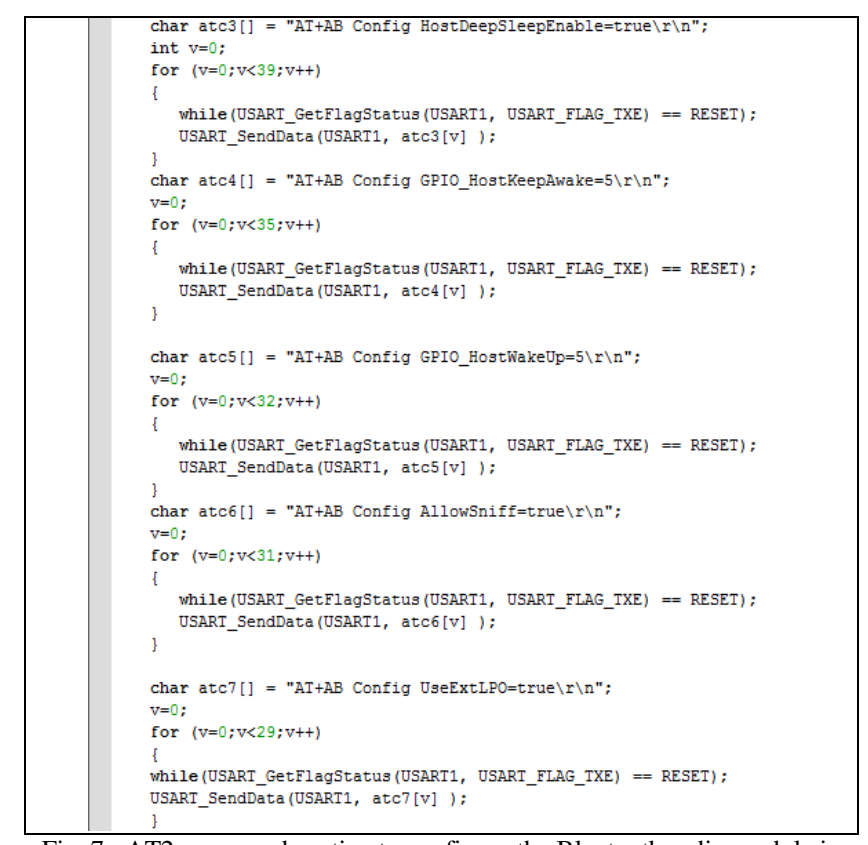

Fig. 7- AT2 command routine to configure the Bluetooth radio module in sniff mode.

Further functional improvements and in terms of energy efficiency of device could be the consequence of a meticulous customization of the components to avoid redundant structures or with the replacement of some of them with low-power versions. A first choice may be the replacement of the Bluetooth module with only a specific BLUETOOTH and FM transceiver system on chip. The SPBT2632C2A, for example, was chosen mainly for reasons of footprint thanks to is microsize form factor compared to other modules on the market. However the module is constituted mainly by the STLC2690 Bluetooth system on chip and by an ARM Corte STM32 family microcontroller which represents a redundant component source of an additional consumption of current. An optimal solution could be the use of a compact radio Bluetooth low energy solution (typically $6 \mathrm{~mm}$ x $6 \mathrm{~mm}$, WQFN package) that is the latest generation in trade. The STM32F10xx family microcontroller could be also replaced by an STM32Lxx ultra low power family one. Furthermore, an accurate study can be expected regarding the implementation of harvesting solutions for the recovery of power in the battery by exploiting the transduction of kinetic energy into an electrical quantity.

\section{REFERENCES}

[1] G. Van Den Broek, F. Cavallo, C. Wehrmann, "AALIANCE Ambient Assisted Living Roadmap", IOS Press, 2010.

[2] ABI Research, "Home Monitoring for Seniors Will Drive 36 Million Wearable Wireless Device Market", 2012.

[3] Svanberg J.,"Consumer Electronics and Wireless M2M", Berg Insight , September 2013.

[4] Bhas N., "Smart Wearable Device", Junipier Research, October 2103.

[5] "Body Area Networks for Sports and Healthcare", ABI Research, 2012.

[6] Vinjamuri R., Crammond D.J., Kondziolka D., Lee Heung-No, Mao Zhi-Hong, "Extraction of Sources of Tremor in Hand Movements of Patients With Movement Disorders", IEEE Transactions on Information Technology in Biomedicine, Vol. 13, No. 1, Jan 2009.
[7] Huber M., Rabin B., Docan C., Burdea G.C., AbdelBaky M., Golomb M.R., "Feasibility of Modified Remotely Monitored In-Home Gaming Technology for Improving Hand Function in Adolescents With Cerebral Palsy", IEEE Transactions on Information Technology in Biomedicine, March 2010,Vol. 14, No. 2, p.526-34.

[8] Choi Y., "Ubi-REHAB: An android-based portable augmented reality stroke rehabilitation system using the eGlove for multiple participants". 2011, International Conference on Virtual Rehabilitation. Rehab Week Zurich, ETH Zurich Science City, Switzerland, June 27-29, 2011.

[9] Mohd Ali A. M., Ambar R., Abdul Jamil M. M., Mohd Wahi A. J., Salim S., "Artificial Hand Gripper Controller via Smart Glove for Rehabilitation Process", 2012 International Conference on Biomedical Engineering (ICoBE).

[10] Amma C., Georgi M., Schultz T., "Airwriting: Hands-free Mobile Text Input by Spotting and Continuous Recognition of 3d-Space Handwriting with Inertial Sensors", ISWC, Newcastle, 18-22 June 2012, p.52-59.

[11] Niazmand K., Tonn K., Kalaras A., Fietzek U.M., Mehrkens J.H., Lueth T.C., "Quantitative Evaluation of Parkinson's Disease using sensor based smart Glove",CBMS, Bristol, UK, 27-30 Jun 2011, pp.1-8.

[12] Vutinuntakasame S., Jaijongrak V., Thiemjarus S. "An Assistive Body Sensor Network Glove for Speech- and Hearing- Impaired

[13] Disabilities", BSN, Dallas, TX, 23-25 May 2011, pp.7-12.

[14] Kim J.W., Lee J.H, Kwon Y., Kim C.S, Eom G.M, Koh S.B, Kwon D.Y, Park K.W. "Quantification of bradykinesia during clinical finger taps using a gyrosensor in patients with Parkinson's disease". Med. Biol. Eng. Comput. 2011, 49: 365-371.

[15] Mera T.O., Heldman D.A., Espay A.J., Payne M., Giuffrida J.P., "Feasibility of home-based automated Parkinson's disease motor assessment". Journal of Neuroscience Methods, 2012, 203: 152-156.

[16] Dai H., Otten B., Mehrkens J.H., D' Angelo L. T., Lueth T.C., "A Novel Glove Monitoring System Used to Quantify Neurological Symptoms During Deep-Brain Stimulation Surgery", IEEE SENSORS JOURNAL, VOL. 13, NO. 9, SEPTEMBER 2013

[17] Dai H.,Otten B.,Mehrkens J.H., D'Angelo L.T., Lueth T.C., "A Novel Glove Monitoring System Used to Quantify Neurological Symptoms During Deep-Brain Stimulation Surgery", IEEE Sensors Journal, Vol.13, No.9, Sept 2013.

[18] Web site: www.kickstarter.com/projects/1761670738/ring-shortcuteverything? ref=discovery.

[19] Akshay D., Anam K., Surekha K.S., "Magic Glove- Wireless Hand Gesture Hardware Controller", IEEE Electronics and Communication Systems (ICECS), 2014.

[20] M. Weilguni, W. Smetana, G. Radosavlevic, J. Nicolics, A. Hofmann, W. Goebl ,"A ring-shaped LTCC/HTCC sensor for detection of finger forces in clarinet playing", Sensors, 2012 IEEE.

[21] Cavallo F., Esposito D., Rovini E., Aquilano M., Carrozza M.C., Dario P., Maremmani C., Bongioanni P. "Preliminary evaluation of SensHand V1 in assessing motor skills performance in Parkinson disease," Rehabilitation Robotics (ICORR), 2013 IEEE International Conference on , vol., no., pp.1,6, 24-26 June 2013.

[22] Cavallo F., Maremmani C., Esposito D., Rovini E., Aquilano M., Dario P., Carrozza M.C, "Not-glove inertial sensor system for movement analisys of hand: SensHand V1", Italian patent, 14/01/2013.

[23] Cavallo F., Maremmani C., Esposito D., Rovini E., Aquilano M., Dario P., Carrozza M.C., "Inertial rings-based sensor system for movement analisys of hand: SensHand V2", Italian patent, 14/01/2013.

[24] Cavallo F., Maremmani C., Esposito D., Rovini E., Aquilano M., Dario P., Carrozza M.C., "Method and related apparatus for monitoring biomechanical performances of human limbs". PCT/IB2014/058265, 14 Jan 2014. 